\title{
Volatile Chemical Interaction Between Undamaged Plants: Effects at Higher Trophic Levels
}

\author{
Robert Glinwood
}

\begin{abstract}
This chapter discusses whether plant chemical communication is a mechanism by which plant genetic diversity can affect the natural enemies of herbivores. Plant genetic diversity influences natural enemies, and these insects use volatile chemical cues to locate suitable habitats. However, the importance of chemical communication for these interactions has not been considered. In this chapter, the latest research on chemical communication between undamaged plants is reviewed. Evidence for the fact that volatile chemical communication between weeds and barley, and between different barley genotypes, can influence host plant and habitat selection by aphid natural enemies, polyphagous ladybirds and parasitic wasps, is presented. The results suggest that aphid natural enemies may recognise the effects of plant-plant volatile interaction and volatile mixing as cues denoting favourable habitats. This represents a new aspect of the ecology of plant communication that may be exploited for sustainable plant protection.
\end{abstract}

\section{Introduction}

Plants and insects have to overcome many challenges to survive and reproduce and need to respond to information in their environment. Chemical information is abundant in the habitats in which plants and insects live, and while we have known for a long time that insects use chemical cues from other insects and plants, it took longer to establish that plants also use volatile chemical signals in ways that appear to be adaptive. It is now generally accepted that plants respond to defence-inducing volatile signals released after attack by herbivores (Chapter 'Within-Plant Signalling by Volatiles Triggers Systemic Defences'), and their informational role is gradually

\footnotetext{
R. Glinwood

Department of Ecology, Swedish University of Agricultural Sciences, 75007 Uppsala, Sweden e-mail: robert.glinwood@ekol.slu.se
} 
being revealed (Heil and Bueno 2007; Halitschke et al. 2008; Chapter 'Within-Plant Signalling by Volatiles Triggers Systemic Defences'). However, plants also respond to chemicals released by undamaged plants (e.g. Runyon et al. 2006).

In allelopathy, for example, plant substances that escape into the environment may affect the growth and development of neighbours (Rice 1984). Although allelopathy is an important issue in agricultural science, affecting many aspects of plant coexistence and competition (Weston and Duke 2003), investigation of its effects at higher trophic levels such as herbivores and their natural enemies has started only recently (Ninkovic et al. 2006). The natural enemies of herbivores respond to habitat cues denoting prey abundance and quality. Specialised natural enemies, such as parasitic wasps, use cues indicating the presence of their hosts (Vet and Dicke 1992), whereas polyphagous predators respond to more general indicators of habitat quality (Pettersson et al. 2005). The vegetational composition of habitats is therefore of great relevance to natural enemies.

There is growing interest in the effects of plant diversity on higher trophic levels. In many cases, increased plant genetic diversity has been found to influence herbivore natural enemies in a positive way (Root 1973; Russell 1989; Cook et al. 2007). Given that these insects are often well developed in their use of chemical cues, and that they must be able to recognise plant genetic diversity in order to respond, it is surprising that the role of chemical interactions is only now starting to receive attention. In this chapter I ask the question 'is plant chemical communication a mechanism by which plant genetic diversity can affect herbivore natural enemies?' I review evidence from investigations on plant volatile interactions affecting aphids on barley, which has opened an exciting new perspective on the ecology of plant communication.

\section{Increased Plant Genetic Diversity Affects Higher Trophic Levels}

\subsection{Between Species Diversity}

A concept emerging from the study of ecosystem function is that multitrophic interactions can be directly affected by plant biodiversity (Hooper et al. 2005). The greatest focus has been on increased diversity of species, which has been found to support higher diversity and abundance of both herbivorous and predatory arthropod species (Siemann et al. 1998; Haddad et al. 2001). Much of the classic work on plant diversity in relation to agricultural systems relates to mixed species intercropping, and has led to the development of a number of theories explaining how complex plant habitats suppress pest insects and benefit their natural enemies (Root 1973; Russell 1989; Andow 1991). These theories, while not universally proven, have been supported by experiments in agricultural (Andow 1991) and natural ecosystems (e.g. Koricheva et al. 2000; Haddad et al. 2001; Otway et al. 2005). 
Despite extensive research on the effects of plant diversity at higher trophic levels, understanding of the mechanistic background to these responses is still limited. Plants sharing a common environment can interact chemically with each other, and can contribute to the combined chemical profile of the habitat. However, whereas chemical mechanisms have been mentioned among theories seeking to explain the effects of mixed cropping on herbivores and natural enemies (e.g. Uvah and Coaker 1984), the role of direct chemical interaction between plants has not been widely considered.

\subsection{Within-Species Genotype Diversity}

Emphasis on the importance of plant species diversity for trophic interactions may have hidden the fact that diversity between different genotypes of the same plant species has its own ecological significance. Due to the hierarchical structure of trophic food webs, it has been pointed out that plant traits specifically influencing natural enemies should be relatively fewer than those affecting their herbivore prey. It has therefore been assumed that variation resulting from combining genotypes of a single plant species should have only a weak affect at the third trophic level (Vinson 1999; Johnson and Agrawal 2005). This view appears to be changing, and evidence for effects of within species genotype diversity on higher trophic levels is starting to accumulate. For example, Johnson (2008) found that plant genotype diversity can directly affect the abundance of insect predators in a natural ecosystem. However, the strongest effects of plant genotype diversity may be found in managed crop systems rather than natural ones because, against the relatively homogenous genetic background typical of most agricultural monocultures, genetic diversity within the plant species may be more apparent. This also opens the possibility to exploit insect responses for sustainable crop protection.

In fact, mixing different genotypes of the same species of crop plant has been shown to reduce the incidence of damage-causing organisms that use the plants as hosts (Power 1991; Mundt 2002; Ninkovic et al. 2002; Cadet et al. 2007). Again, it is not known to what extent chemical signalling is involved in these interactions. However, the role of chemical signalling as a mechanism contributing to the impact of plant genotype interactions at higher trophic levels has been more extensively studied in a model system consisting of barley, aphids and their natural enemies, and this work will be described next.

\section{Volatile Interactions Between Undamaged Plants Affect Higher Trophic Levels}

It has become apparent that chemical interaction between undamaged plants is a complex process that affects trophic interactions beyond the plant level (Glinwood et al. 2003; Ninkovic et al. 2006), and may even have an informational value for the 
receiving plant. This has been shown in interactions between barley and weeds and between different genotypes of barley itself. Barley exposed to volatiles from undamaged plants of a different cultivar adjusts its biomass allocation to favour roots rather than shoots, a response that may indicate increased readiness for competition with a neighbouring plant (Ninkovic 2003). Barley also responds to volatiles from weeds and other barley genotypes by becoming less acceptable as a host plant to aphid herbivores (Pettersson et al. 1999; Ninkovic et al. 2002; Glinwood et al. 2004; Glinwood et al. 2007). These interactions are reviewed in Chapter 'Volatile Interactions Between Undamaged Plants: Effects and Potential for Breeding Resistance to Aphids'.

These findings have led to the introduction of the term, allelobiosis to describe the wider trophic effects of plant interaction via chemicals (Pettersson et al. 2003; Ninkovic et al. 2006). The three key aspects of the definition of allelobiosis are (1) the chemical interaction occurs between undamaged plants, (2) the interaction may be beneficial for the receiving plant and (3) the responses of the receiving plant affect organisms at other trophic levels. Aspect 1 separates allelobiosis from a large body of research on interplant signalling, which focuses on signals released by infected/infested plants, while aspect 3 separates allelobiosis from the plantfocused approach of allelopathy.

Volatile interaction between undamaged plants can also affect the third trophic level. There are two main mechanisms by which this may occur, outlined in Fig. 1. First by allelobiosis (Fig. 1a), in which direct volatile interaction between neighbouring plants induces responses in the receiving plant that affect its interactions with natural enemies, via either volatile emission or quality of the herbivore prey. Secondly, if there is variation in the volatile profiles of the different plant genotypes, they can combine to give an increased volatile chemical diversity that natural enemies could use as an indicator of habitat quality (Fig. 1b). The evidence for these different mechanisms in the barley model system will be discussed next, focusing separately on interactions between different plant species and between genotypes of the same species.

\subsection{Allelobiosis Between Different Plant Species}

The seven spot ladybird Coccinella septempunctata is a polyphagous predator that uses aphids as a high quality food source (Hodek and Honek 1996). It is commonly found in barley fields where it is an important member of the complex of natural enemies that regulate aphid populations. Experiments with adult $C$. septempunctata, barley and two common weeds, Elytrigia repens and Cirsium arvense have demonstrated how both allelobiosis and plant volatile diversity may support the habitat preferences of adult $C$. septempunctata in barley.

Surveys in a barley field showed that adult ladybirds occurred in significantly greater numbers in patches containing either E. repens or $C$. arvense than in weedless patches (Ninkovic and Pettersson 2003). The plant biomass was similar 

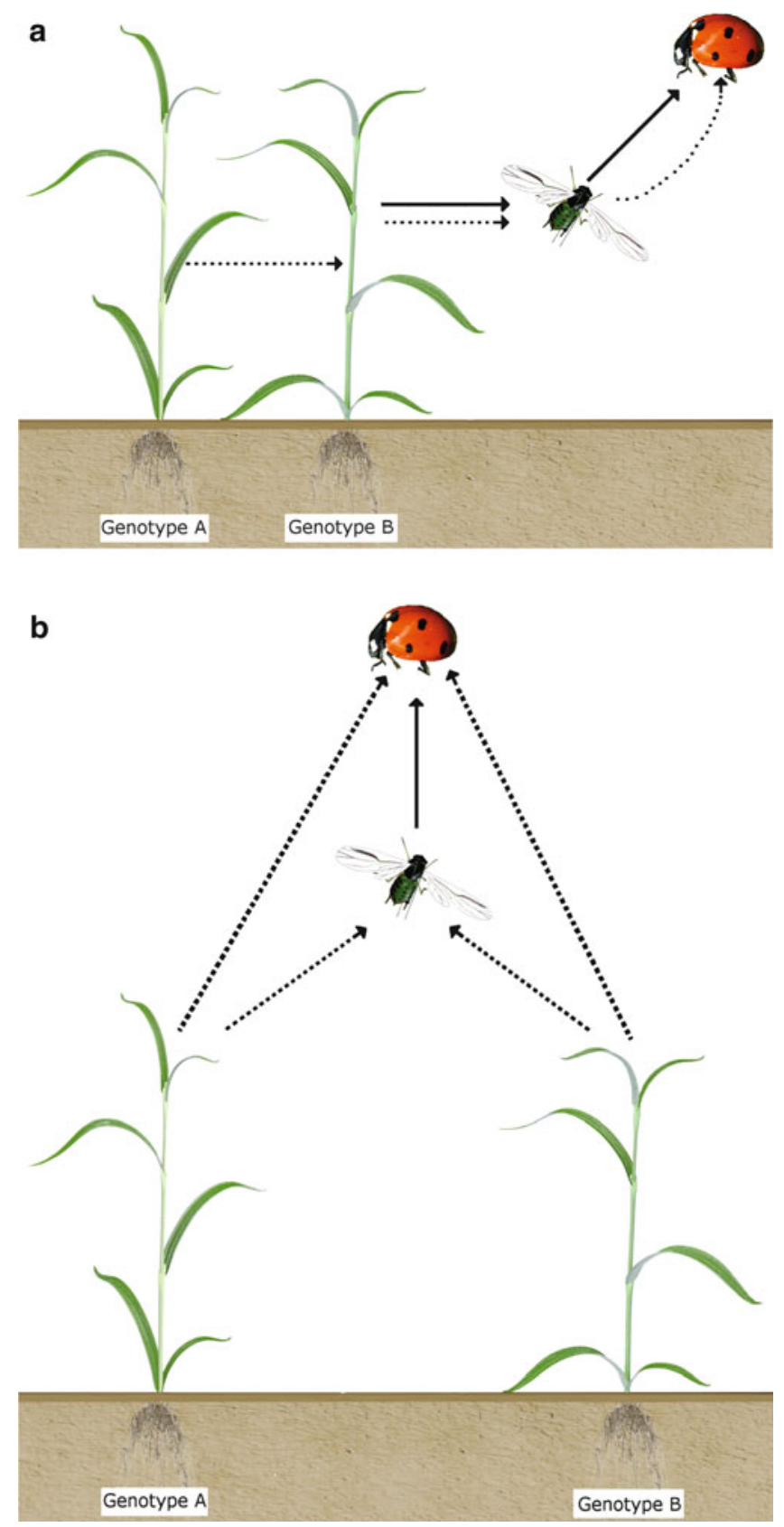

Fig. 1 a and b. Two mechanisms by which mixing different plant genotypes may affect the natural enemies of herbivores via volatile chemicals (dotted lines) and host quality (bold lines). In (a) volatiles from one plant genotype induce responses in a second genotype that affect plant volatile emission and herbivore host quality. In (b) mixed odours of the different plant genotypes affect natural enemy orientation 
in the different patches, the weeds were not flowering and there were no aphids in the crop. This suggests that the ladybird distribution represented a habitat preference influenced by the presence of the weeds. In a series of laboratory experiments, the importance of plant volatile cues for the observed habitat preference was investigated (Ninkovic and Pettersson 2003). Volatiles of neither weed were directly attractive to ladybirds, however mixed odours of either weed together with barley were more attractive than the odour of barley alone. When the E. repens plants were removed from the system, barley plants that had been exposed to volatiles from the weed were not more attractive than unexposed barley, suggesting that volatile mixing was responsible for the previously observed attraction. However, barley plants that had been exposed to volatiles from $C$. arvense continued to be attractive to ladybirds even after the weed had been removed.

This suggests the involvement of allelobiosis, in which weed volatiles induced changes in the volatile profile of the exposed barley plants that made them more attractive to ladybirds. Interestingly, barley exposed to volatiles from this weed were found to be less acceptable to aphids both on contact and via their odour (Glinwood et al. 2004; Chapter 'Volatile Interaction Between Undamaged Plants: A Short Cut to Coexistence'), indicating that effects of allelobiosis occur over at least two trophic levels.

Aphids are attacked by small parasitic wasps in the subfamily Aphidiinae, and these too are considered important members of the aphid natural enemy complex. Allelobiosis between weeds and barley also affects olfactory orientation by these insects. While odour of the thistle Cirsium vulgare was repellent to female Aphidius ervi, barley that had been exposed to volatiles from $C$. vulgare became significantly more attractive than unexposed plants (Fig. 2). Presumably the thistle itself represents a non-host plant for a parasitoid that has been reared on a cereal-cereal aphid system, while attraction to exposed barley suggests induced changes that modify the volatile profile.

The responses of ladybirds in the experiments described above are consistent with theories on the positive effects of plant diversity on natural enemies (Root 1973; Bach 1980; Russell 1989) and also support previous studies on the importance of plant composition for the ladybird habitat preference (Leather et al. 1999; Elliott et al. 2002). They add a new perspective to the theoretical debate, namely that chemical interaction between plants, either directly by allelobiosis or via odour mixing, may comprise a mechanism by which insects detect plant diversity.

\subsection{Allelobiosis Between Different Genotypes of the Same Plant Species}

Several studies have shown that allelobiosis between different cultivars of barley affects both plant biomass allocation and interaction with aphid herbivores 
a

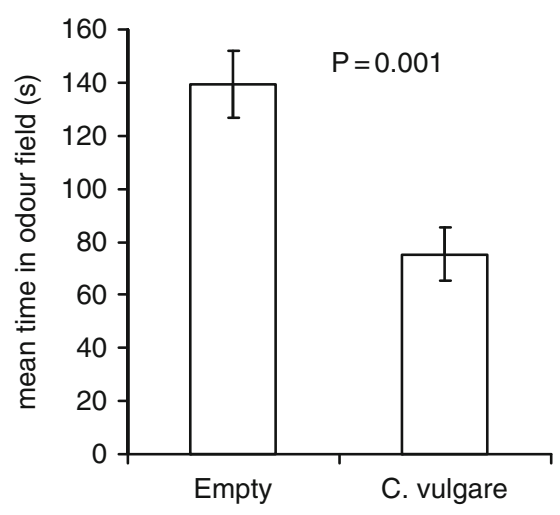

b

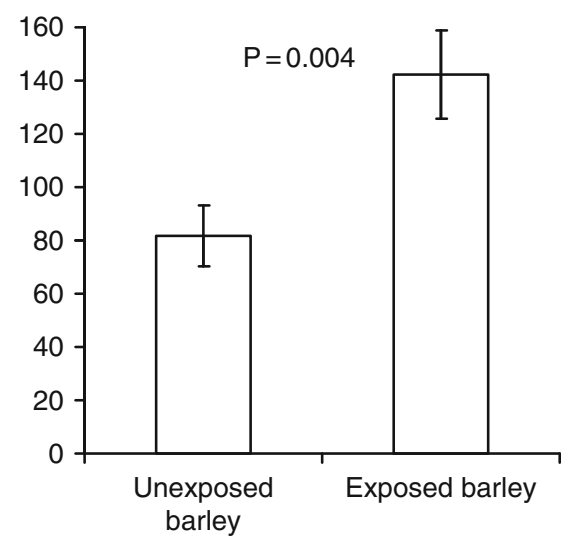

Fig. 2 Olfactory preference of the aphid parasitoid Aphidius ervi in a two-way olfactometer given a choice between (a) odour from the thistle Cirsium vulgare and clean air or (b) odour of barley previously exposed to volatiles from $C$. vulgare and unexposed barley. Values of $\mathrm{P}$ from Wilcoxon tests

(Ninkovic 2003; Ninkovic et al. 2002; Glinwood et al. 2007; Ninkovic and Åhman 2009; Chapter 'Volatile Interactions Between Undamaged plants: Effects and Potential for Breeding Resistance to Aphids'). As is the case for weed-barley interactions, volatile exposures between barley cultivars also affect aphid natural enemies. The effects appear to follow the same pattern as found for aphids, with only specific cultivar combinations influencing natural enemy foraging behaviour. In a field experiment, significantly more ladybird adults were recorded in stands of a mixture of two particular barley cultivars than in stands of either cultivar alone (Ninkovic et al. unpublished). Laboratory experiments showed that, although neither cultivar was more attractive, volatile exposure of one cultivar to the other caused the odour of exposed plants to become more attractive to ladybirds. When the odours of the cultivars were combined, this same cultivar combination was also more attractive than the odour of either cultivar alone. This is the first evidence that direct chemical interaction and odour mixing in genotypes of the same plant species can affect olfactory orientation by a polyphagous predator.

These findings are backed up by results with further barley cultivar combinations, in which attraction of ladybirds persisted in exposed plants for up to 7 days after the end of the exposure period, suggesting long term changes in plant status that are reflected by the profile of volatile emission (Glinwood et al. 2009). Interestingly there was a close agreement between those cultivar combinations that gave both negative effects on aphid plant acceptance and positive effects on ladybird attraction, suggesting involvement of a common mechanism. Aphid parasitoids of the species Aphidius colemani were also attracted to the odour of exposed plants from these combinations. 
The adaptive significance of these insect responses is still not apparent, however aphid natural enemies are known to be sensitive to the quality of their aphid hosts, and should forage optimally to locate and exploit the best quality hosts. Barley plants that became attractive to aphid natural enemies after volatile exposure also appear to be less preferred as hosts by aphids. However, experiments suggest that aphids developing on plants exposed to volatiles from a different cultivar represent higher quality hosts for aphid natural enemies. For example, ladybirds consumed more aphids that had developed on exposed plants than on unexposed plants, and female parasitoids attacked and laid more eggs in aphids that had developed on exposed plants (Glinwood et al. 2009). Parasitoid egg development did not appear to be affected, suggesting that natural enemies may have handled aphid prey items more efficiently, perhaps due to decreased defensive behaviour by the aphids.

In addition to the effects of allelobiosis, odour mixing from different barley cultivars affects ladybird olfactory orientation. The combined odours of certain pair wise combinations of barley cultivars were more attractive to ladybirds than an equivalent biomass of either individual cultivar alone (Fig. 3). Interestingly the cultivar combinations that gave the clearest effects via mixed odours were those in which one cultivar became more attractive after exposure to volatiles from the other. The more specialised aphid parasitoids, however, did not show a clear pattern of attraction to mixed odours.
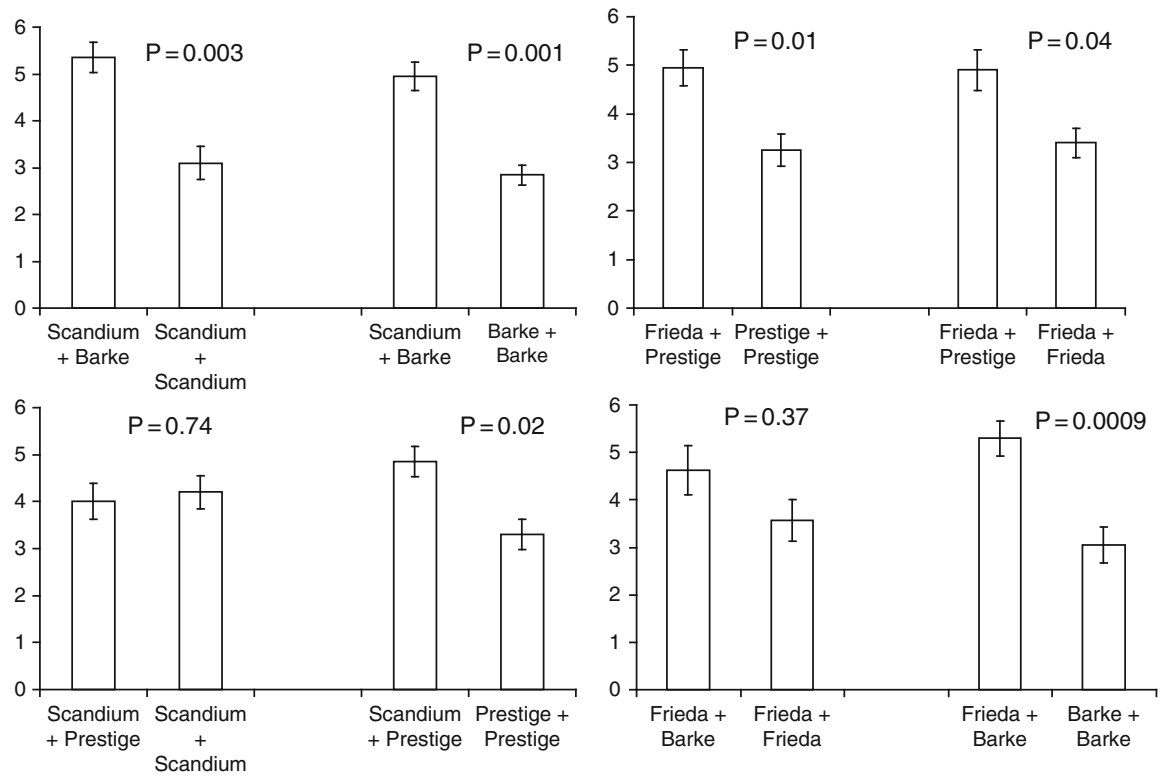

Fig. 3 Olfactory preference of the seven spot ladybird Coccinella septempunctata in a two-way olfactometer, given a choice between mixed odours of two different barley cultivars and odour of an equal biomass of each cultivar alone. Mean no. observations in odour field. Values of $\mathrm{P}$ from Wilcoxon tests (Glinwood et al. (2009), with kind permission of Springer Science and Business Media) 


\section{Does Plant Genetic Diversity Affect Natural Enemies Via Volatile Interaction and Diversity?}

The findings on chemical interaction in barley reviewed here give a new perspective on one possible route linking plant and natural enemy levels. For a polyphagous predator like $C$. septempunctata, high quality habitats are characterised by the availability of a range of different food sources (Elliott et al. 2002; Pettersson et al. 2008). So, while aphids are a required food for this species, other small insects and pollen are also important and increased plant diversity should thus be favourable. However in order to respond to plant diversity, insects must be able to detect it. The work on barley has shown that chemical interactions between undamaged plants can make them more attractive to aphid natural enemies. It is still not clear what changes are induced in barley plants exposed to allelobiosis. It is possible that changes in the odour profile of exposed plants result from physiological events connected with growth and biomass allocation (Ninkovic 2003) or are coupled to a general stress condition induced by the presence of a close neighbour (Chapter 'Volatile Interaction Between Undamaged Plants: A Short Cut to Coexistence').

In either case, these volatile cues may enable natural enemies to locate habitats or patches with a favourable degree of plant diversity. They may then benefit from more diverse and abundant food in the case of ladybirds, or be able to forage more efficiently due to the condition of their prey in the case of both ladybirds and parasitoids. C. septempunctata also responded positively to the combined odours of weeds and barley and certain mixtures of barley cultivars. These responses may represent a further mechanism by which ladybirds can detect a favourable level of increased plant genetic diversity. Aphid parasitoids did not respond to the odour mixtures as strongly as ladybirds, which is expected considering their higher degree of specialisation.

These hypotheses seem more appropriate for the interactions between weeds and barley than for those between different barley genotypes, since variation in plant characteristics is presumably much lower within species than between species. However, if ladybirds can detect indicators of plant genetic diversity through volatile cues, the behavioural responses mediating this may be triggered by relatively small increases in odour diversity against a homogenous background, as is the case in agricultural monocrops such as cereals. Enhancement of natural enemies in mixed cropping has been explained by the provisioning of alternative resources (Root 1973) but it is unlikely that cultivars of the same plant species would fulfil this role for a generalist predator such as $C$. septempunctata, so whether these responses are adaptive for ladybirds foraging in barley is still unclear and needs to be investigated. Nevertheless, the work discussed here opens the way for further investigation on how chemical cues communicate plant genetic diversity to higher trophic levels. 


\section{Conclusions}

We are now beginning to understand the wider aspects of plant volatile chemical signalling. The ecological role of damage-induced cues is becoming clear (Halitschke et al. 2008; Chapter 'Within-Plant Signalling by Volatiles Triggers Systemic Defences') and explanations for the adaptive significance of induced volatile emission are emerging (Heil and Ton 2008). Our understanding of the broader implications for chemical signalling between undamaged plants is, however, still in its infancy. The current chapter has proposed a mechanism contributing to the effects of plant genetic diversity at the natural enemy level, while the work reviewed in Chapters 'Volatile Interaction Between Undamaged Plants: A Short Cut to Coexistence' and 'Volatile Interactions Between Undamaged Plants: Effects and Potential for Breeding Resistance to Aphids' suggests these interactions can be exploited for insect pest management. Although most of the knowledge is limited to a model system with barley, there is increasing evidence from agricultural systems that mixing different genotypes of the same plant species can affect organisms that use the plants as hosts.

Research on these questions should continue along two closely coordinated lines:

1. Investigation of the ecosystem role of chemical signalling between undamaged plant individuals within habitats and between plant habitats and insects. Chemical interaction and chemical diversity may be previously unconsidered mechanisms linking genetic diversity at the plant level with behaviour and population dynamics at the primary and secondary consumer levels. Since even polyphagous insects have a specific set of nutritional requirements, the strongest effects are expected to occur in combinations of plant genotypes with particular characteristics, rather than with continuous increases in plant diversity per se. This is reflected in the research on barley in which effects at higher trophic levels occur only in particular genotype combinations.

2. Exploitation of these interactions by using mixed cropping to manipulate pest herbivores and their natural enemies in agricultural systems (Exploiting Plant Signals in Sustainable Agriculture). Although intercropping with different plant species is long established, it may be possible to achieve similar results by mixing genotypes of the same plant species, giving a more practical approach to sustainable plant protection.

We are beginning to view plant chemical communication as a natural facet of the behaviour of plants under normal conditions and not only as a response to attack. Key questions to be answered include whether this occurs commonly in natural or managed ecosystems and whether it represents a genuine exchange of information between plants. Further study is likely to reveal fascinating new aspects of the ecology of plant communication. 


\section{References}

Andow DA (1991) Vegetational diversity and arthropod population responses. Annu Rev Entomol 36:561-586

Bach CE (1980) Effect of plant diversity and time of colonization on an herbivore-plant interaction. Oecologia 44:319-326

Cadet P, Berry SD, Leslie GW, Spaull VW (2007) Management of nematodes and a stalk borer by increasing within-field sugarcane cultivar diversity. Plant Pathol 56:526-535

Cook SM, Khan ZR, Pickett JA (2007) The use of push-pull strategies in integrated pest management. Annu Rev Entomol 52:375-400

Elliott NC, Kieckhefer RW, Michels GJ, Giles KL (2002) Predator abundance in alfalfa fields in relation to aphids, within-field vegetation, and landscape matrix. Environm Entomol 31:253-260

Glinwood RT, Pettersson J, Ninkovic V, Ahmed E, Birkett M, Pickett JA (2003) Change in acceptability of barley plants to aphids after exposure to allelochemicals from couch-grass (Elytrigia repens). J Chem Ecol 29:259-272

Glinwood R, Ninkovic V, Ahmed E, Pettersson J (2004) Barley exposed to aerial allelopathy from thistles (Cirsium spp.) becomes less acceptable to aphids. Ecol Entomol 29:188-195

Glinwood RT, Gradin T, Karpinska B, Ahmed E, Jonsson LMV, Ninkovic V (2007) Aphid acceptance of barley exposed to volatile phytochemicals differs between plants exposed in daylight and darkness. Plant Signal Behav 2:205-210

Glinwood R, Ahmed E, Qvarfordt E, Ninkovic V, Pettersson J (2009) Airborne interactions between undamaged plants of different cultivars affect insect herbivores and natural enemies. Arthropod-Plant Interact 3:219-224

Haddad NM, Tilman D, Haarstad J, Ritchie M, Knops JMH (2001) Contrasting effects of plant richness and composition on insect communities: a field experiment. Am Nat 158:17-35

Halitschke R, Stenberg JA, Kessler D, Kessler A, Baldwin IT (2008) Shared signals - 'alarm calls' from plants increase apparency to herbivores and their enemies in nature. Ecol Lett 11:24-34

Heil M, Bueno JCS (2007) Within-plant signaling by volatiles leads to induction and priming of an indirect plant defense in nature. Proc Natl Acad Sci USA 104:5467-5472

Heil M, Ton J (2008) Long-distance signalling in plant defence. Trends Plant Sci 13:264-272

Hodek I, Honek A (1996) Ecology of coccinellidae. Kulwer, Dordrecht

Hooper DU, Chapin FS III, Ewel JJ, Hector A, Inchausti P, Lavorel S, Lawton JH, Lodge DM, Loreau M, Naeem S, Schmid B, Setälä H, Symstad AJ, Vandermeer J, Wardle DA (2005) Effects of biodiversity on ecosystem functioning: a consensus of current knowledge. Ecol Monogr 75:3-35

Johnson MTA, Agrawal AA (2005) Plant genotype and environment interact to shape a diverse arthropod community in evening primrose (Oenothera biennis). Ecology 86:875-885

Johnson MTA (2008) Bottom-up effects of plant genotype on aphids, ants, and predators. Ecology 89:145-154

Koricheva J, Mulder CPH, Schmid B, Joshi J, Huss-Danell K (2000) Numerical responses of different trophic groups of invertebrates to manipulations of plant diversity in grasslands. Oecologia 125:271-282

Leather SR, Cooker RCA, Fellowes MDE, Rombe R (1999) Distribution and abundance of ladybirds (Coleoptera: Coccinellidae) in non-crop habitats. Eur J Entomol 96:23-27

Mundt CC (2002) Use of multiline cultivars and cultivar mixtures for disease management. Annu Rev Phytopathol 40:381-410

Ninkovic V (2003) Volatile communication between barley plants affects biomass allocation. J Exp Bot 54:1931-1939

Ninkovic V, Åhman I (2009) Aphid acceptance of Hordeum genotypes is affected by plant volatile exposure and is correlated with aphid growth. Euphytica 169:177-185 
Ninkovic V, Pettersson J (2003) Searching behaviour of sevenspotted ladybird, Coccinella septempunctata - effects of plant-plant odour interaction. Oikos 100:65-70

Ninkovic V, Olsson U, Pettersson J (2002) Mixing barley cultivars affects aphid host plant acceptance in field experiments. Entomol Exp Appl 102:177-182

Ninkovic V, Glinwood R, Pettersson J (2006) Communication between undamaged plants by volatiles: the role of allelobiosis. In: Baluška F, Mancuso S, Volkmann D (eds) Communication in plants: neuronal aspects of plant life. Springer-Verlag, Berlin, pp 421-434

Otway SJ, Hector A, Lawton JH (2005) Resource dilution effects on specialist insect herbivores in a grassland biodiversity experiment. J Anim Ecol 74:234-240

Pettersson J, Ninkovic V, Ahmed E (1999) Volatiles from different barley cultivars affect aphid acceptance of neighbouring plants. Acta Agric Scand Sect B 49:152-157

Pettersson J, Ninkovic V, Glinwood R (2003) Plant activation of barley by intercropped conspecifics and weeds: allelobiosis. BCPC Crop Sci Technol 2:1135-1144

Pettersson J, Ninkovic V, Glinwood R, Birkett MA, Pickett JA (2005) Foraging in a complex environment - semiochemicals support searching behaviour of the seven spot ladybird. Eur J Entomol 102:365-370

Pettersson J, Ninkovic V, Glinwood R, Al Abassi S, Birkett M, Pickett J, Wadhams L (2008) Chemical stimuli supporting foraging behaviour of Coccinella septempunctata L (Coleoptera: Coccinellidae): volatiles and allelobiosis - a minireview. Appl Entomol Zool 43:315-321

Power AG (1991) Virus spread and vector dynamics in genetically diverse plant populations. Ecology 72:232-241

Rice EL (1984) Allelopathy, 2nd edn. Academic, New York

Root RB (1973) Organization of plant-arthropod association in simple and diverse habitats: The fauna of collards (Brassica oleraceae). Ecol Mon 43:95-124

Runyon JB, Mescher MC, Moraes CMD (2006) Volatile chemical cues guide host location and host selection by parasitic plants. Science 313:964-1967

Russell EP (1989) Enemies hypothesis: a review of the effects of vegetational diversity on predatory insects and parasitoids. Environ Entomol 18:590-599

Siemann E, Tilman D, Haarstad J, Ritchie M (1998) Experimental tests of the dependence of arthropod diversity on plant diversity. Am Nat 152:738-750

Uvah III, Coaker TH (1984) Effect of mixed cropping on some insect pests of carrots and onions. Entomol Exp Appl 36:159-167

Vet LEM, Dicke M (1992) Ecology of infochemical use by natural enemies in a tritophic context. Annu Rev Entomol 37:141-172

Vinson SB (1999) Parasitoid manipulation as a plant defense strategy. Ann Ent Soc Am 92:812-828

Weston LA, Duke SO (2003) Weed and crop allelopathy. Crit Rev Plant Sci 22:367-389 